\title{
Brain energetics plays a key role in the coordination of electrophysiology, metabolism and hemodynamics: evidence from an integrated computational model
}

\author{
G Capo Rangel $^{1} \quad$ J Prezioso $^{2} \quad$ L Gerardo-Giorda $^{1} \quad$ E Somersalo $^{2} \quad$ D Calvetti ${ }^{2}$ \\ ${ }^{1}$ Basque Center for Applied Mathematics \\ ${ }^{2}$ Department of Mathematics, Applied Mathematics and Statistics \\ Case Western Reserve University
}

\begin{abstract}
The energetic needs of brain cells at rest and during elevated neuronal activation has been the topic of many investigations where mathematical models have played a significant role providing a context for the interpretation of experimental findings. A recently proposed mathematical model, comprising a double feedback between cellular metabolism and electrophysiology, sheds light on the interconnections between the electrophysiological details associated with changes in the frequency of neuronal firing and the corresponding metabolic activity. We propose a new extended mathematical model comprising a three-way feedback connecting metabolism, electrophysiology and hemodynamics. Upon specifying the time intervals of higher neuronal activation, the model generates a potassium based signal leading to the concomitant increase in cerebral blood flow with associated vasodilation and metabolic changes needed to sustain the increased energy demand. The predictions of the model are in good qualitative and quantitative agreement with experimental findings reported in the literature, even predicting a slow after-hyperpolarization of a duration of approximately 16 seconds matching experimental observations.
\end{abstract}

\section{Introduction}

Understanding the connections between electrophysiology, metabolism and hemodynamics in human brain is essential for shedding light on the mechanisms behind their mutual regulation, and for elucidating to what extent a disruption in one of these cerebral functions may, partly or completely, impair the others [1,2]. A deeper understanding of the delicate interplay between these three systems would help also appropriately establish how reliably measurements from brain imaging modalities following hemodynamic changes can be used to infer on brain electrophysiology $[3,4,5,6]$.

It is well-known that increase in neuronal activity is followed by higher cerebral blood flow in the surrounding region [7], however a comprehensive explanation for the phenomenon is still missing [8,9]. Another question waiting for a definitive answer is how the brain meets the energetic costs of membrane depolarization and repolarization in connection with excitatory or inhibitory neuronal activity, a topic that continues to motivate research on brain energy metabolism $[10,11]$. Over the past two decades, astrocytes have been increasingly recognized as having a 
much more prominent role in brain energy metabolism than initially believed, elevating them to major metabolic players in the energetics of excitation and inhibition when it comes to the cycling of neurotransmitters, maintaining redox balance, and clearing potassium following neuronal firing [12, 13, 14, 15]. In computational neuroscience, the paradigm based on networks of neurons alone has begun to shift towards a more integrated picture, where astrocytes have a more pronounced role through their control of ion concentrations in extracellular space, in turn affecting neuronal firing. Furthermore, it has been demonstrated that astrocyte end feet enclose brain capillary and may play a role in cerebral blood flow regulation, although the underlying mechanism connecting it to the neuronal activation is not fully understood yet $[9,16,17]$. A predictive mathematical model that can account for all three cerebral functions and their mutual interactions is a useful tool for testing possible feedback modes.

Predictive cell level mathematical models have been deemed necessary to advance the understanding of the human neurovascular complex in the absence of direct observations. Following the original contribution of Hodgkin and Huxley [18], neuronal firing has been extensively studied by means of mathematical models of different complexity, including models describing the dynamics of ion fluxes responsible for transmission of action potentials across synaptic clefts $[19,20,21]$. As computational neuroscience moves towards integrating more neurophysiology into models of neuronal firing, the energetic costs of operating the sodium potassium pump have started being accounted for [22]. A recent mathematical model [23], validated by experimental findings in the literature, has successfully included a two-way feedback process between neuronal activation and cerebral metabolism.

This paper proposes a new computational predictive integrated model of the hemodynamics, metabolism and electrophysiology of a human neurovascular unit comprising neuron, astrocyte and arterial-venous vascular compartments, that can be used to investigate in silico the regulation of metabolic and vascular response to neuronal activity in human brain. In previous models combining energy metabolism and electrophysiology, the delivery of metabolic substrates through blood flow is defined as an input variable and adjusted to meet the energetic needs of the neuronal activity. Conversely, previous models for hemodynamics assume an external input signal controlling the compliance of the blood vessels, without a direct coupling to energy consuming activities. The novelty of this article is the introduction of a double feedback loop between these two modeling regimes: In the proposed model, the blood vessel compliance is controlled by the extracellular potassium level, which is a function of the neuronal activity, while the metabolic system must rely on metabolite delivery through the blood flow, and the state of the electro-metabolic complex depends on their availability. This coupled model may help understand observed phenomena that continue to be the topic of active investigation, including the unexplained overshoot of oxygen delivery during neuronal activation known as the oxygen paradox, some diseased states such as migraine and cortical spreading depression, and the neuronal damage following prolonged epileptic seizures. Computed experiments show that the time courses of membrane potential, metabolite concentrations and blood flow predicted by the model are in qualitative and quantitative agreement with experimental results reported in the literature, a remarkable finding since the changes in metabolism, hemodynamics and ion fluxes are determined internally by the model, not specified as input. Figure 1 shows a schematic view of the interaction between metabolism, electrophysiology, and hemodynamics under two scenarios, at low activity level, referred to as resting state, and during high neuronal activation. 

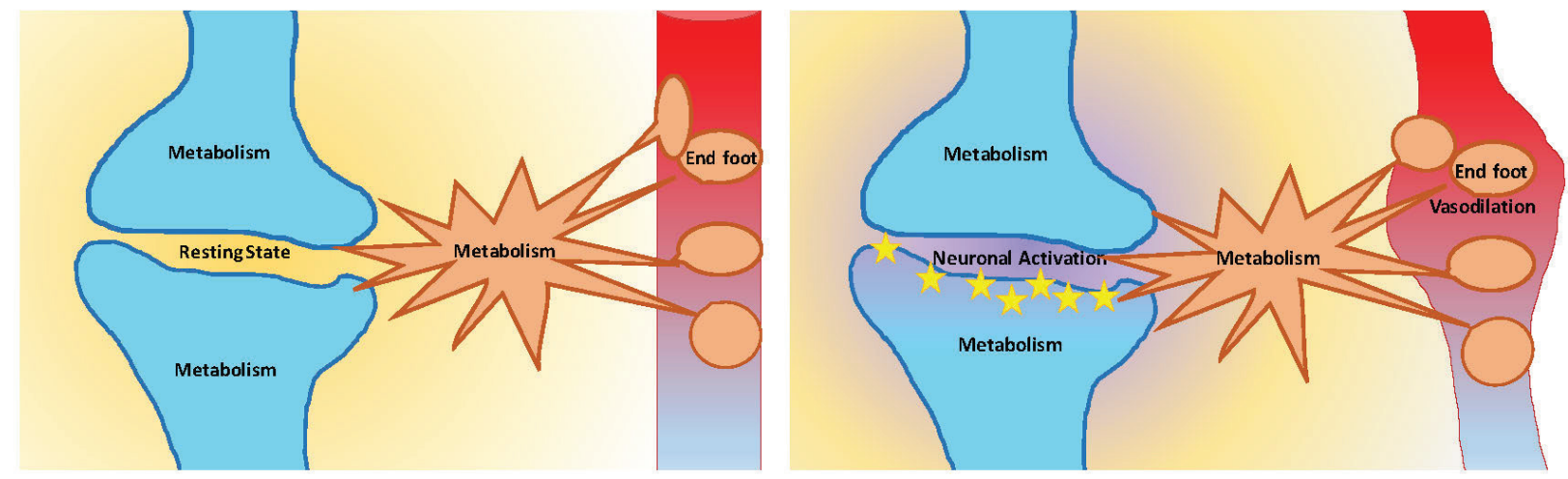

Figure 1: A schematic of the integrated hemodynamic-electro-metabolic model. The neuron (blue) and astrocyte (orange) are assigned separate compartments, but there is no division between pre- and post-synaptic neuron, nor between soma, neuronal dendrites and axons, or astrocyte end feet. A separate compartment is reserved for blood, accounting for arterial, capillary, and venous components in the hemodynamic module. In line with current understanding, we assume that the extra blood volume rushing to the activation site in response to the activation is due to vasodilation in the arterioles triggered by the activation induced increased potassium concentration in extracellular space (ecs). The uniform yellow background in the left panel indicates the higher $\mathrm{Na}^{+}$concentration in extracellular space at rest: in the right panel the lilac cloud illustrates the temporary increase in extracellular $\mathrm{K}^{+}$concentration during the repolarization phase due to the $\mathrm{Na}^{+}-\mathrm{K}^{+}$pump activation, also responsible for the increase in energy demand, symbolically denoted by yellow stars.

Before presenting the details of the model, we discuss briefly the interpretation of the model, and in particular, how the phenomena described in different spatial scales should be understood.

The metabolic and hemodynamic moduls used in our integrated model are spatially homogenized lumped models, and the parameter values describing reaction rates and metabolite transports between compartments are scaled to correspond to a volume equivalent to one gram of gray matter tissue. If $\left|\Omega_{0}\right|$ denotes the reference volume corresponding to one gram tissue, and $|\Omega|$ is the volume of the brain tissue sample $\Omega$ that we want to model, and $[M]$ is the vector of the metabolite concentration, the dynamic model, whose details are discussed in the forthcoming sections, is of the form

$$
\rho \frac{d[M]}{d t}=F([M]), \quad \rho=\frac{|\Omega|}{\left|\Omega_{0}\right|},
$$

where $F$ is a vector whose components represent the parametric reaction and transport fluxes in the system, with parameters scaled for the reference volume. This equation reveals that the model can be rescaled back to the reference volume by scaling the time $t$ by a factor proportional to the volume. Alternatively, if we want to adjust the metabolic model to correspond to the volume of few neurons, we could scale the reaction and transport rate parameters in the dynamic model dividing by the volume ratio $\rho$. Scaling the metabolic model down to few neurons' scale, on the other hand, would make the neuron-astrocyte geometry play a role, including the position with respect to the metabolite supply by blood flow, while the lumped model paradigm is an average model assuming that fine details are ignored. Likewise, using too large volumes violates the basic assumptions of well-mixed models, since diffusion starts to play a significant role. Instead of attempting to perform a geometric scaling of the model with an ill-defined volume of few neurons and astrocytes, we retain the metabolic model as is, and instead adjust the electrophysiology model so that it corresponds to a neuron population of one gram tissue. This model matching is not straightforward, and is discussed at length in [23]. 
The electrophysiological description is based on extensions of the classical Hodgkin-Huxley model to comprise the ion transport between neurons and astrocytes. We therefore interpret that the electrophysiological neuron-astrocyte model in fact represents a neuron-astrocyte population through a model of an average neuron, surrounded by supporting astrocytes. A characteristic measure used here for describing the level of neuronal activity is the neuronal firing rate. In particular, consider a volume containing a neuron population, each neuron firing at a given time instant with its own frequency $f$. Let $p(f)$ denote the frequency distribution over the neuron population at a given time,

$$
\int_{0}^{\infty} p(f) d f=1 .
$$

In practice, there is an absolute cut-off maximum frequency $f_{\max }$, determined by the duration of the refractive period after depolarization of the membrane, hence $p(f)=0$ for $f>f_{\max }$. The average firing frequency of the population is defined as

$$
\bar{f}=\int_{0}^{\infty} f p(f) d f .
$$

To account for the total energy need of the neuron-astrocyte complex included in the volume under consideration, we model the energy consumption by assuming that the volume contains neurons firing with average frequency $\bar{f}$ over a time period that corresponds to the time scales of the metabolic and hemodynamic units, that is, in the scale of minutes. Observe that a single neuron does not have to sustain a fixed activity over the whole period; it is enough to assume that the distribution $p$ remains unaltered over the time interval. Therefore, a single neuron in the population may undergo a series of active bursting periods and longer resting periods. Our model does not provide detailed information about single neurons, although we will discuss the model predictions also from the point of view of single neurons.

\section{Materials and Methods}

The novel mathematical model that we are proposing extends a recent model integrating metabolism and electrophysiological activity of neurons and astrocytes [23], combining it with one estimating blood vessels' compliance and blood flow changes in response to a vasodilatory stimulus $[24,25,26]$. In the former, the computational challenge of carrying out time integration in view of the vastly different time scales of metabolism and neuronal firing are addressed by a fast-slow time integration scheme. The proposed method respects the fine time scale nature of electrophysiology by using a refined time discretization to model electrical activity within each step at the metabolic scale, and integrating the net energy demand to inform the coarse scale metabolic model. We refer to the model combining the metabolism and electrophysiological activity as electro-metabolic model. Tracking the related hemodynamic changes adds yet another time scale to the model, which has to be dealt with carefully to avoid numerical instabilities and keep a handle on the computational costs.

Electro-Metabolic Model (EMM) The starting point for the model integrating metabolism and electrophysiological neuron-astrocyte activity is a spatially lumped four-compartment metabolic model comprising neuron (n), astrocyte (a), extracellular space (ecs) and blood (b) compartments, each occupying a specified volume fraction $\eta_{c}, c=$ $\{n, a, b, \mathrm{ecs}\}$. As is customary in the spatially lumped metabolic models, the cellular compartments represent the net metabolic response of a population of cells in a volume unit. In particular, the neuron compartment represents both pre- and postsynaptic neurons. The model parameters and time scales are adjusted to correspond a volume 
of one gram of tissue of gray matter. Following the dynamical metabolic model [27], each cellular compartment is characterized by its own metabolic network and all exchanges of metabolites between them occur through the extracellular space, which is the only compartment to exchange metabolites with the blood through the Blood Brain Barrier (BBB). The model, written in terms of the concentrations of metabolites in each compartment, collected in the time dependent vector

$$
[M](t)=\left[\begin{array}{c}
{[M]_{b}(t)} \\
{[M]_{\mathrm{ecs}}(t)} \\
{[M]_{n}(t)} \\
{[M]_{a}(t)}
\end{array}\right],
$$

tracks the dynamics of glucose, lactate and oxygen in all compartments and, in neuron and astrocyte, pyruvate, creatine, phosphocreatine, ATP, ADP, NADH and $\mathrm{NAD}^{+}$, for a total of 26 species, taking into account the compartmental multiplicity of each species. The biochemical reactions included in the model are listed in Table 1.

\begin{tabular}{|l|l|}
\hline Name & Chemical Reaction \\
\hline Glycolysis & $\mathrm{Glc}+2 \mathrm{NAD}+2 \mathrm{ADP} \longrightarrow 2 \mathrm{Pyr}+2 \mathrm{NADH}+2 \mathrm{ATP}$ \\
Lactate dehydrogenase 1 & $\mathrm{Pyr}+\mathrm{NADH} \longrightarrow \mathrm{Lac}+\mathrm{NAD}^{+}$ \\
Lactate dehydrogenase 2 & $\mathrm{Lac}+\mathrm{NAD}^{+} \longrightarrow \mathrm{Pyr}+\mathrm{NADH}$ \\
Tricarboxylic acid cycle & $\mathrm{Pyr}+\mathrm{ADP}+5 \mathrm{NAD}+3 \mathrm{CO}_{2}+\mathrm{ATP}+5 \mathrm{NADH}$ \\
Oxidative phosphorylation & $\mathrm{O}_{2}+2 \mathrm{NADH}+5 \mathrm{ADP} \longrightarrow 2 \mathrm{NAD}^{+}+5 \mathrm{ATP}+2 \mathrm{H}_{2} \mathrm{O}$ \\
Phosphocreatine dephosphorylation & $\mathrm{PCr}+\mathrm{ADP} \longrightarrow \mathrm{Cr}+\mathrm{ATP}$ \\
Creatine phosphorylation & $\mathrm{Cr}+\mathrm{ATP} \longrightarrow \mathrm{PCr}+\mathrm{ADP}$ \\
\hline
\end{tabular}

Table 1: Biochemical reactions in the metabolic model.

Each reaction contributes a reaction flux in the system. Reaction fluxes are modeled using a Michaelis-Menten type kinetics, equipped with regulation by the phosphorylation and redox state of the cell,

$$
p_{c}=\frac{[\mathrm{ATP}]_{c}}{[\mathrm{ADP}]_{c}}, r_{c}=\frac{[\mathrm{NADH}]_{c}}{\left[\mathrm{NAD}^{+}\right]_{c}}, \quad \text { for } c=\{\mathrm{n}, \mathrm{a}\} .
$$

Because all communication between neuron and astrocyte is assumed to occur through the extracellular space, changes in glucose, lactate and oxygen concentrations in the latter depend on the transfer fluxes of these metabolites between extracellular space and neuron or astrocyte, as well as on the rate at which metabolites are exchanged with the blood compartment.

In the blood compartment, the changes over time of metabolite concentrations depend on blood flow $(\mathrm{CBF}(t))$, mixing ratio between venous and arterial blood, assumed constant, and the exchange rate of metabolites between blood and extracellular space, which for glucose and lactate are expressed in a symmetric Michaelis-Menten form, while for oxygen it follows a modified Fick's law. The arterial concentrations $[M]_{\text {art }}$ and the blood flow are considered as an input for the metabolic system. The two cellular compartments are equipped with the biochemical reactions involved with either the production or the consumption of ATP: the complete list of biochemical reactions accounted for in our model, and the mathematical form of the corresponding fluxes can be found in the literature [27]. Here our focus is on the important role of ATP in coupling the electrophysiological activity and metabolism, in particular on ATP dephosphorylation, ATP $\rightarrow$ ADP $+\mathrm{P}_{i}$, releasing $E=30.5 \mathrm{~kJ} / \mathrm{mol}$ of energy that can be used to meet the energetic needs of the sodium-potassium pump in neuron, the various energetic needs of astrocyte participating in 
glutamine-glutamate cycling and potassium cleaning, and general household maintenance in both cells. Therefore, the reaction rates of ATP dephosphorylation, denoted by $\psi_{\text {ATPase }}^{n}$ in neuron and $\psi_{\text {ATPase }}^{a}$ in astrocyte, implicitly converted into energy flux by the factor $E$, are not modeled in terms of metabolite concentrations but rather in terms of the energetic costs of the sodium-potassium pump inducing the ion mass current $I_{\text {pump }}$, glial potassium uptake of the astrocyte resulting in the ion current $I_{\text {glia }}$, the energetic cost incurred by the glutamate-glutamine cycle induced sodium current $I_{\mathrm{Glu}}$, as well as a variety of household tasks in neuron and astrocyte, denoted by $H_{n}$ and $H_{a}$ respectively. More specifically,

$$
\begin{aligned}
& \psi_{\text {ATPase }}^{n}=H_{n}+s\left(\eta_{n} I_{\text {pump }}+0.33 I_{\mathrm{Glu}}\right), \\
& \psi_{\text {ATPase }}^{a}=H_{a}+s\left(\frac{\eta_{\mathrm{ecs}}}{2} I_{\text {glia }}+2.33 I_{\mathrm{Glu}}\right),
\end{aligned}
$$

where $\eta_{n}$ and $\eta_{\text {ecs }}$ are the volume fractions of the neuronal and extracellular space compartments, $H_{n}$ and $H_{a}$ account for unspecified household energy in neuron and astrocyte, and $s$ is a proportionality constant. The parameter values used in our computed experiment are listed in Table 2; for a detailed discussion on how the values were chosen, we refer to the original article [23]. Accounting for the volume fractions of each compartment via the diagonal volume matrix D,

$$
\mathrm{D}=\left[\begin{array}{cccc}
\eta_{\mathrm{b}} \mathrm{I}_{3} & 0 & 0 & 0 \\
0 & \eta_{\mathrm{ecs}} \mathrm{I}_{3} & 0 & 0 \\
0 & 0 & \eta_{\mathrm{n}} \mathrm{I}_{10} & 0 \\
0 & 0 & 0 & \eta_{\mathrm{a}} \mathrm{I}_{10}
\end{array}\right]
$$

where $\mathrm{I}_{k}$ is the identity matrix of size $k \times k$, and the integer $k$ denotes the number of metabolites in the compartment, we can write the governing equations of the metabolism as

$$
\mathrm{D} \frac{d[M]}{d t}=F\left([M],[M]_{\text {art }}, \psi_{\text {ATPase }}^{\mathrm{n}} \psi_{\text {ATPase }}^{\mathrm{a}}, \mathrm{CBF}\right) .
$$

In the equations governing neuronal firing, which are a modification $[19,21,20]$ of the original formulation by Hodgkin and Huxley [18], the rate of change of the membrane voltage potential (V) in time is directly proportional to the currents of sodium $I_{\mathrm{Na}}$, potassium $I_{\mathrm{K}}$ and chloride $I_{\mathrm{Cl}}$, and inversely proportional to the membrane capacitance $C$,

$$
\frac{d V}{d t}=-\frac{1}{C}\left(I_{\mathrm{Na}}+I_{\mathrm{K}}+I_{\mathrm{Cl}}\right)
$$

The ionic currents are functions of the gating variables $(n, m, h)$, the voltage and their corresponding conductance and equilibrium potentials $\left(V_{\mathrm{Na}}, V_{\mathrm{K}}, V_{\mathrm{Cl}}\right)$,

$$
\begin{aligned}
I_{\mathrm{Na}} & =g_{\mathrm{Na}}\left[m_{\infty}(V)\right]^{3} h\left(V-V_{\mathrm{Na}}\right)+g_{\mathrm{Na}, \text { leak }}\left(V-V_{\mathrm{Na}}\right), \\
I_{\mathrm{K}} & =g_{\mathrm{K}} n^{4}\left(V-V_{\mathrm{K}}\right)+g_{\mathrm{K}, \text { leak }}\left(V-V_{\mathrm{K}}\right), \\
I_{\mathrm{Cl}} & =g_{\mathrm{Cl}}\left(V-V_{\mathrm{Cl}}\right),
\end{aligned}
$$

where $g_{\mathrm{Na}}, g_{\mathrm{K}}, g_{\mathrm{Cl}}$ are the ion conductances, and the leak conductances and $n$ and $h$ the activation and inactivation gating functions of potassium and sodium, respectively. The gating variable $m_{\infty}(V)$ is assumed to be in equilibrium, while the variables $h$ and $n$ satisfy the standard Hodgkin-Huxley equations. The reversal potentials $V_{i}$ are computed with the Nernst equation. Unlike in the original Hodgkin-Huxley model, the intracellular and extracellular concen- 
trations are not constants, but change due to the sodium/potassium pump action, astrocytic potassium cleaning, and diffusion as

$$
\begin{aligned}
& \frac{d\left[\mathrm{~K}^{+}\right]_{\mathrm{ecs}}}{d t}=\gamma \beta I_{\mathrm{K}}-2 \beta I_{\mathrm{pump}}-I_{\mathrm{glia}}-I_{\mathrm{diff}}, \\
& \frac{d\left[\mathrm{Na}^{+}\right]_{\mathrm{i}}}{d t}=-\gamma \beta I_{\mathrm{Na}}-3 I_{\mathrm{pump}}
\end{aligned}
$$

where $\beta$ denotes the ratio between the volume fraction of the neuron and the volume fraction of the extracellular space, while $\gamma$ converts the electric current into a mass flux [19,21].

Two additional algebraic conditions connect the intra- and extracellular concentrations,

$$
\begin{aligned}
{\left[\mathrm{K}^{+}\right]_{\mathrm{i}} } & =140 \mathrm{mM}+\left(10.5 \mathrm{mM}-\left[\mathrm{Na}^{+}\right]_{\mathrm{i}}\right), \\
{\left[\mathrm{Na}^{+}\right]_{\mathrm{ecs}} } & =144 \mathrm{mM}-\beta\left(\left[\mathrm{Na}^{+}\right]_{\mathrm{i}}-10.5 \mathrm{mM}\right) .
\end{aligned}
$$

In accordance with what was proposed in the literature [23], the expression for the sodium-potassium pump in terms of the concentrations of sodium and potassium and the strength of the sodium potassium pump ( $\left.\rho_{\text {pump }}\right),[19,21,20]$, is modified to account for ATP availability by adding a dependency on the phosphorylation state $p_{\mathrm{n}}$, defined in (2) as the ratio between the concentration of adenosine triphosphate and adenosine diphosphate,

$$
I_{\text {pump }}=\frac{p_{\mathrm{n}}}{\mu_{\text {pump }}+p_{\mathrm{n}}}\left(\frac{\rho_{\text {pump }}}{\left.1+\exp \left(25-\left[\mathrm{Na}^{+}\right]_{\mathrm{i}}\right) / 3\right)}\right)\left(\frac{1}{1+\exp \left(2.2-\left[\mathrm{K}^{+}\right]_{\mathrm{ecs}}\right.}\right),
$$

where $\mu_{\text {pump }}$ is an affinity constant. Similarly, the expression for the glial potassium clearing is

$$
I_{\text {glia }}=\frac{p_{\mathrm{a}}}{\mu_{\text {glia }}+p_{\mathrm{a}}}\left(\frac{G_{\text {glia }}}{\left.1+\exp \left(14.7-\left[\mathrm{K}^{+}\right]_{\mathrm{ecs}}\right) / 2.5\right)}\right),
$$

where $G_{\text {glia }}$ is the uptake strength of the glia, $\mu_{\text {glia }}$ is an affinity constant and $p_{\text {a }}$ denotes phosphorylation state in astrocyte. We remark that the formulas (9) and (10) are as in [23], but the parameters, listed in Table 2, have been modified so that the potassium levels predicted by the model are in line with the physiological values reported in literature [28]. In line with models proposed in the literature [14, 29], potassium diffusion is governed by extracellular potassium concentration, a diffusion coefficient $\epsilon$ and the surrounding potassium concentration $k_{s}$,

$$
I_{\text {diff }}=\epsilon\left(\left[\mathrm{K}^{+}\right]_{\mathrm{ecs}}-k_{s}\right)
$$

The units and values of all parameters appearing in the expressions above for the baseline frequency of $4 \mathrm{~Hz}$ characteristic for the neuron at rest are listed in Table 2. The details concerning the choice of parameters can be found in the literature [23]. Higher neuronal frequencies are triggered by a stimulus parameter $\xi$ which causes the leak conductances of both sodium and potassium to temporarily increase,

$$
\begin{aligned}
g_{\text {Na,leak }} & =(1+\xi(t)) g_{\text {Na,leak }}^{0}, \\
g_{\mathrm{K}, \text { leak }} & =(1+\xi(t)) g_{\mathrm{K}, \text { leak }}^{0}
\end{aligned}
$$

This way of inducing neuronal activation, proposed by several authors [23, 30, 31], is motivated by the large influx of sodium and potassium accompanying the increase in glutamate during activation. In our computed experiments, 
we model an awake resting state as corresponding to a frequency of $8 \mathrm{~Hz}$, attained by setting the stimulus parameter to $\xi=0.05$.

The sodium leak current induced by the increase of the membrane permeability through the function is given by

$$
I_{\mathrm{Na}, \text { leak }}=g_{\mathrm{Na}, \text { leak }}\left(V-V_{\mathrm{Na}}\right)
$$

while the average sodium leak current during resting state is denoted by $I_{\mathrm{Na} \text {,leak }}^{0}$.

The glutamate-glutamine flux is accounted for through

$$
I_{\mathrm{Glu}}=\left\{\begin{array}{ll}
\frac{\gamma}{\sigma}\left(I_{\mathrm{Na}, \text { leak }}-I_{\mathrm{Na}, \text { leak }}^{0}\right) & \text { if } \xi(t)>0 \\
0 & \text { if } \xi(t)=0
\end{array},\right.
$$

which relates it to the energetic cost of glutamate-glutamine cycling during synaptic activity [23].

We collect the five unknowns of the electrophysiological model in the vector

$$
u(t)=\left[\begin{array}{c}
V(t) \\
{\left[\mathrm{Na}^{+}\right]_{\mathrm{i}}(t)} \\
{\left[\mathrm{K}^{+}\right]_{\mathrm{ecs}}(t)} \\
n(t) \\
h(t)
\end{array}\right] \in \mathbb{R}^{5}
$$

and express the governing equations as the nonlinear system of ordinary differential equations

$$
\frac{d u}{d t}=f\left(u, p_{\mathrm{n}}, p_{\mathrm{a}}, \xi\right)
$$

where the input phosphorylation states are determined by the metabolic model described above.

The Hodgkin-Huxley model and generalizations thereof were originally thought for a single cell, while the metabolic spatially lumped model represents a volume containing an unspecified size of cell population. When integrating neuronal firing with metabolism and hemodynamics, we implicitly assume the electrophysiological model to represent the activity of an average neuron-astrocyte pair. Therefore the energetic needs of the complex are the integrated needs of the cell population, with the electrophysiological single cell model providing the justification for the coupling between the energetics of neuron and astrocyte populations. Summarizing, the input for the EMM consists of

- Arterial concentrations of glucose, oxygen and lactate $[M]_{\mathrm{art}}, M=\{$ glucose, oxygen, lactate $\}$;

- Cerebral Blood Flow $\mathrm{CBF}(t)$;

- Activation function $\xi(t)$.

The metabolic and electrophysiological modules are connected through a double feedback loop where

- The metabolic model defines $p_{\mathrm{n}}(t)$ and $p_{\mathrm{a}}(t)$, the inputs for the electrophysiological model; 
- The electrophysiological model determines the ATP demand used to compute $\psi_{\text {ATPase }}^{n}$ in neuron and $\psi_{\text {ATPase }}^{a}$ in astrocyte, comprising the input for the metabolic model.

The difference of several orders of magnitude in the characteristic time scales of metabolism and electrophysiology poses a real challenge for computing model predictions with a numerical time integrator, the millisecond range of electrophysiology time scale requiring a much finer time discretization than what would suffice for the metabolism, whose time scale is in the order of seconds or minutes. A numerical scheme designed specifically to accommodate the different time scales has been recently proposed [23].

\begin{tabular}{|c|c|c|c|c|c|c|c|c|}
\hline \multicolumn{3}{|c|}{ Electrophysiology model } & \multicolumn{3}{|c|}{ Metabolic model } & \multicolumn{3}{|c|}{ Hemo-Electro-Metabolic model } \\
\hline Symbol & Value & Unit & Symbol & Value & Unit & Symbol & Value & Unit \\
\hline$g_{\mathrm{Na}}$ & 46 & $\mathrm{mS} / \mathrm{cm}^{2}$ & {$[\mathrm{Glc}]_{\text {art }}$} & 5 & $\mathrm{mM}$ & $\alpha$ & 100 & - \\
\hline$g_{\mathrm{K}}$ & 16.25 & $\mathrm{mS} / \mathrm{cm}^{2}$ & {$[\mathrm{Lac}]_{\text {art }}$} & 1.1 & $\mathrm{mM}$ & $K^{\text {base,max }}$ & 2.995 & $\mathrm{mM}$ \\
\hline$g_{\mathrm{Cl}}$ & 0.05 & $\mathrm{mS} / \mathrm{cm}^{2}$ & {$\left[\mathrm{O}_{2}\right]_{\text {art }}$} & 9.14 & $\mathrm{mM}$ & & & \\
\hline$g_{\text {Na. leak }}^{0}$ & 0.0175 & $\mathrm{mS} / \mathrm{cm}^{2}$ & $\mathrm{CBF}$ & 0.4 & $\mathrm{~mL} / \mathrm{min}$ & & & \\
\hline$g_{\mathrm{K} \text {,leak }}^{0}$ & 0.02 & $\mathrm{mS} / \mathrm{cm}^{2}$ & $\eta_{n}$ & 0.4 & - & & & \\
\hline $\begin{array}{l}{ }_{\mathrm{N}, \text { leak }} \\
\gamma\end{array}$ & 0.0445 & $\mathrm{mMcm}^{2} / \mu \mathrm{C}$ & $\eta_{a}$ & 0.3 & - & & & \\
\hline$\sigma$ & 103 & - & $\eta_{\text {ecs }}$ & 0.3 & - & & & \\
\hline$C$ & 1 & $\mu \mathrm{F} / \mathrm{cm}^{2}$ & $\eta_{\mathrm{b}}$ & 0.04 & - & & & \\
\hline$\varphi$ & 100 & $\mathrm{msec}^{-1}$ & $H_{n}$ & 4.30 & $\mathrm{mM} / \mathrm{min}$ & & & \\
\hline$k_{s}$ & 3 & $\mathrm{mM}$ & $H_{a}$ & 3.58 & $\mathrm{mM} / \mathrm{min}$ & & & \\
\hline$\rho$ & 13.83 & $\mathrm{mM} / \mathrm{sec}$ & & & & & & \\
\hline$G_{\text {glia }}$ & 20.75 & $\mathrm{mM} / \mathrm{sec}$ & & & & & & \\
\hline$\varepsilon$ & 9.33 & $\mathrm{sec}^{-1}$ & & & & & & \\
\hline$\mu_{\text {pump }}$ & 0.1 & - & & & & & & \\
\hline$\mu_{\text {glia }}$ & 0.1 & - & & & & & & \\
\hline
\end{tabular}

Table 2: Parameters for the Hemo-Electro-Metabolic model

\section{Blood flow model}

Neuronal activation is followed by a rapid focal increase of CBF and cerebral blood volume (CBV), supplying the activated region of the brain with the nutrients necessary to support the increased metabolic activity, most importantly oxygen. While experiments confirm the basic mechanism [7], details concerning the underlying signaling are not fully known. Experimental evidence for potential causes of the vasodilation supports different possible mechanisms, including increased concentration of lactate [32], extracellular potassium [33], or nitric oxides originating from the neurotransmission related glutaminase [34]. Evidence points to a central role of astrocytic regulation through the end feet in the signal transmission to the hemodynamic complex $[9,16,17]$.

The increase in CBF and CBV can be understood in terms of activity-induced relaxation of the smooth muscles in the walls of arterial and venous blood vessels and/or pericytes in capillary walls, physically leading to an increased vessel compliance, although it has not been settled definitely which segment of the cerebrovascular network is responsible for the increase of the blood flow; a comprehensive discussion of the topic has been recently published [9]. Recent evidence from optical imaging that the increase in CBF is mainly due to arterial rather than venous blood vessels, made obsolete the hypothesis of a mainly venous contribution of the original balloon [35] and windkessel models [36, 37]. The hemodynamic portion of our integrated model does not exclude some venous and capillary 
contribution, but implicitly assumes a much more prominent arterial contribution, in agreement with recent findings. Although the blood flow has been incorporated in some metabolic models to explain the BOLD signal as a causal response to increased metabolic activity [38], and to connect it to oxygen dynamics in tissue [39, 40, 41], an integrated model including a feedback loop between metabolism and blood flow seems to be missing. In this work, we propose a model interfacing the EMM with a multi-compartment cerebral blood flow model [26] that divides the vascular complex in arterial $(j=1)$, capillary $(j=2)$ and venous $(j=3)$ compartments, each characterized by time dependent volume $v_{j}$, resistance $r_{j}$ and compliance $c_{j}$. Denoting by $f_{j-1}$ and $f_{j}$ the blood flows in and out of the $j$ th compartment, it follows from the conservation of mass that

$$
\frac{d v_{j}}{d t}=f_{j-1}-f_{j}, \quad 1 \leq j \leq 3
$$

The compliance relates the pressure $P_{j}$ in the compartment relative to the intracranial pressure with the compartment volume,

$$
v_{j}=c_{j} P_{j}, \quad 1 \leq j \leq 3,
$$

and by dividing the resistance of each compartment symmetrically between inflow and outflow, the pressure drop between the entry point and exit point is related to the blood flow and resistance,

$$
p_{j}-P_{j}=\frac{1}{2} \frac{r_{j}}{f_{j-1}}, \quad P_{j}-p_{j+1}=\frac{1}{2} \frac{r_{j}}{f_{j}}
$$

where $p_{j}$ is the entry pressure, $p_{j+1}$ the exit pressure.

The resistance of the compartment depends on the dilation of the vascularization, which in turn depends on the volume. Assuming a Poisseuille flow model in a fictitious cylindrical tube, resistance can be expressed in terms of volume,

$$
r_{j} \propto \frac{1}{v_{j}^{2}}
$$

where $\propto$ means proportionality with a proper scaling. Finally, following [26], in order for the compliance to take into account the viscoelastic and stiffness properties of the veins, we write

$$
c_{j}=c_{j}^{*}\left(\frac{k_{j}-\frac{v_{j}}{v_{j}^{*}}}{k_{j}-1}-E_{j} \frac{d v_{j}}{d t}+s_{j}(t)\right), \quad 1 \leq j \leq 3
$$

where $c_{j}^{*}$ and $v_{j}^{*}$ are the baseline compliance and volume, respectively, $k_{j}$ is the stiffness, $E_{j}$ is the viscoelastic constant and $s_{j}=s_{j}(t)$ is the vasodilatory stimulus associated with the compartment. The system can be interpreted in terms of an analog RC circuit with known electromotive force corresponding to the known pressure drop over the full circuit, see Figure 2. 


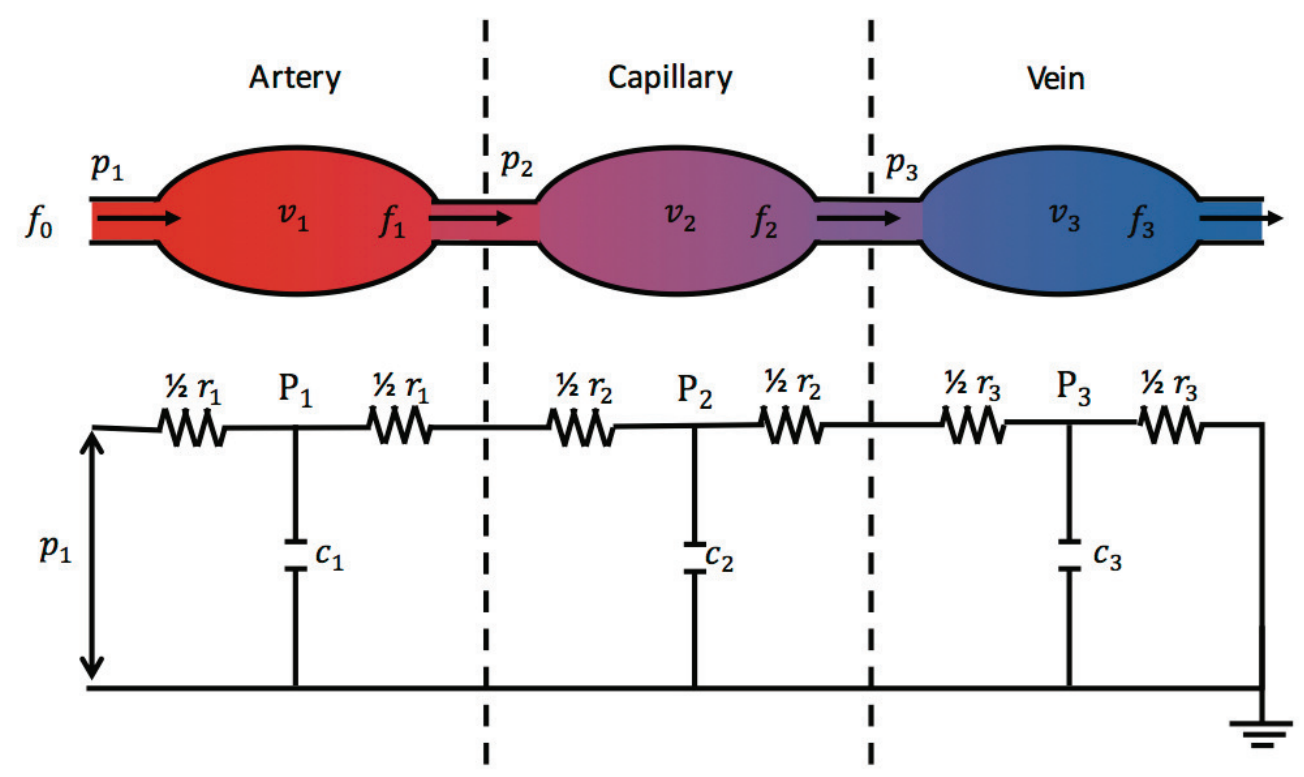

Figure 2: Schematic of the hemodynamic model, and the corresponding analog RC circuit, in which the volumes $\left(v_{j}\right)$ correspond to electric charges, pressures $\left(p_{j}\right)$ to voltages, and blood flows $\left(f_{j}\right)$ to electric currents. The flow resistances $\left(r_{j}\right)$ correspond to electric resistances, and compliances $\left(c_{j}\right)$ to capacitances. The pressures are normalized to intracranial pressure, representing the ground. The given input pressure $p_{1}$ corresponds to the total pressure drop across the complex.

The presence of the derivative of the volume in (19) makes the system a non-standard differential model, and an efficient solution requires careful handling [24]: the cerebral blood flow can be computed as a weighted average over the inter-compartmental flows,

$$
\operatorname{CBF}(t)=\frac{\sum_{j=1}^{3} v_{j}(t)\left[f_{j-1}(t)+f_{j}(t)\right]}{\sum_{j=1}^{3} v_{j}(t)}
$$

To couple the hemodynamic model with the EMM [23], we postulate the following feedback connection mechanisms:

- The blood flow input function of the EMM is computed according to (20) from the output of the hemodynamics model;

- The extracellular potassium concentration $\left[\mathrm{K}^{+}\right]_{\mathrm{ecs}}(t)$ is computed by the EMM and determines the arterial stimulus function $S(t)=s_{1}(t)$ in (19), while $s_{2}=s_{3}=0$. In light of experimental findings [33], we assume that the elevated extracellular potassium concentration triggers a calcium wave in the surrounding astrocytes, which travels along the end feet to the penetrating arterioles, causing an increase in the arterial compliances as described by equation (19). The increased compliance induces an increase in arterial volume (17) and a 
decrease in resistance (18), in turn increasing the downstream pressure leading to an increase of both capillary and venous volumes. The details of the interface are given in the following section.

\section{Coupling Metabolism, Electrophysiology and Blood Flow}

The inputs to the EMM are the arterial metabolite concentrations, blood flow, and the activation function $\xi(t)$ which triggers the neuronal firing. The only input to the hemodynamic model is the vasodilatory stimulus function that we postulate to depend on the extracellular potassium concentration, computed as an output by the EMM, while the CBF is an output of the hemodynamic module. This suggests a double feedback between EMM and hemodynamic model based on the potassium signal and CBF. The only external inputs remaining to be specified are the arterial concentrations and the activation function. A schematic of the complete three-way feedback loop is shown in Figure 3. The dependency of the vasodilatory stimulus on extracellular potassium concentration is modeled as

$$
s(t)=\alpha\left(\left[\mathrm{K}^{+}\right]_{\mathrm{ecs}}-K^{\mathrm{base}, \max }\right) * w(t)=\alpha \int_{0}^{t}\left(\left[\mathrm{~K}^{+}\right]_{\mathrm{ecs}}\left(t^{\prime}\right)-K^{\mathrm{base}, \max }\right) w\left(t-t^{\prime}\right) d t^{\prime}
$$

where $K^{\text {base,max }}$ is the maximum extracellular potassium concentration for awake resting state and $w(t)$ is a weight function such that

$$
w(t)=\exp \left(-\frac{\tau}{\chi}\right), \quad \chi=0.6514 \mathrm{sec}
$$

and $\alpha$ is a volume-dependent scaling constant. Observe that the kernel $w$ describes the fading off of the vasodilatory stimulus after the excess potassium in extracellular space has been removed by astrocyte clearing and diffusion. The time constant $\chi$ is chosen so that after 3 seconds the kernel has decreased to $1 \%$ of its peak value, therefore the length of the interval of integration in (21) can be limited effectively to 3 seconds.

The different characteristic time scales must be handled carefully when designing a numerical scheme to compute model predictions. The time step $\Delta \tau$ of electrophysiology, in the millisecond range, is adaptively chosen by the built-in MATLAB function ode15s; the time step of the metabolic processes is set to $\Delta t=0.05 \mathrm{sec}$, while a time step $\Delta T=1 \mathrm{sec}$ is used for the computation of the much slower changes in blood flow [23]. Denoting by $T_{c}$ the current value in the coarse time step, let $t_{j}=T_{c}+j \Delta t, 0 \leq j \leq m=20$ with $t_{m}=T_{c}+\Delta T$. Denoting by $K_{j}\left(t^{\prime}\right)$ the potassium concentration profile over the interval over $\left[t_{j}, t_{j+1}\right]$, we define

$$
\sigma_{j}=\int_{t_{j}}^{t_{j}+\Delta t}\left(K_{j}\left(t^{\prime}\right)-K^{\text {base,max }}\right) d t^{\prime}
$$

The stimulus function (21) at time $t_{j}$ can now be approximated as

$$
\begin{aligned}
S_{j}=s\left(t_{j}\right) & =\sum_{\ell=1}^{L} \alpha \int_{t_{j}-\ell \Delta t}^{t_{j}-(\ell-1) \Delta t}\left(\left[\mathrm{~K}^{+}\right]_{\mathrm{ecs}}\left(t^{\prime}\right)-K^{\mathrm{base}, \mathrm{max}}\right) w\left(t-t^{\prime}\right) d t^{\prime} \\
& \approx \alpha \sum_{\ell=1}^{L} w(\ell \Delta t) \sigma_{j-\ell}
\end{aligned}
$$


where the entries $\sigma_{j-\ell}$ with negative index refer to entries computed during the previous hemodynamic time steps, and the index $L$ is determined by the condition $w(\ell \Delta) \approx 0$ if $\ell>L$.

The numerical procedure for computing the predictions of the current three-way feedback integrated model of hemodynamics, electrophysiology and metabolism is summarized below. Since the algorithm comprises three different time scales and requires model matching on two time scale interfaces, we divide the algorithm description in two parts, the coarse and the fine time scale marching schemes. Both interfaces use the same algorithmic structure. 


\section{Coarse time scale marching}

Given: Time steps $\Delta t, \Delta T=m \Delta t$;

Initialize: $T_{c}=0, \mathrm{CBF}_{c}=$ baseline value;

while $T_{c}<T_{\max }$ do

Extrapolate $\mathrm{CBF}$ as constant over $\left[T_{c}, T_{c}+\Delta T\right]$. Call it $\mathrm{CBF}^{*}$;

Propagate EEM over $\left[T_{c}, T_{c}+\Delta T\right]$ using $\mathrm{CBF}^{*}$ as input;

Compute $\sigma_{j}, 1 \leq j \leq m$ defined by formula (23), and the stimulus $S_{j}=s\left(T_{c}+j \Delta t\right), 0 \leq j \leq m$;

Propagate the hemodynamic model using $S_{j}$ as input to get updated $\mathrm{CBF}^{+}$at $T_{c}+\Delta T$;

Define $\mathrm{CBF}$ over $\left[T_{c}, T_{c}+\Delta T\right]$ by interpolating linearly between $\mathrm{CBF}_{c}$ and $\mathrm{CBF}^{+}$;

Repropagate EEM over $\left[T_{c}, T_{c}+\Delta T\right]$ using the interpolated $\mathrm{CBF}$ as input;

Update $\mathrm{CBF}_{c}=\mathrm{CBF}^{+}$;

Update $T_{c}=T_{c}+\Delta T$.

end do

Above, we propagate twice the EEM model over a time interval of length $\Delta T$, which is accomplished by running the algorithm below.

\section{Fine time scale marching}

Given: Time steps $\Delta \tau, \Delta t=k \Delta \tau$;

Initialize: $t_{c}=T_{c}, p_{c}=\left(p_{\mathrm{n}}, p_{\mathrm{a}}\right)_{c}=$ values at $T_{c}$;

while $t_{c}<T_{c}+\Delta T$ do

Extrapolate $p=\left(p_{\mathrm{n}}, p_{\mathrm{a}}\right)$ as constant over $\left[t_{c}, t_{c}+\Delta t\right]$. Call it $p^{*}=\left(p_{\mathrm{n}}^{*}, p_{\mathrm{a}}^{*}\right)$;

Propagate electrophysiology model over $\left[t_{c}, t_{c}+\Delta t\right]$ using $p^{*}$ as input;

Compute $\psi=\left[\left(\psi_{\text {ATPase }}^{\mathrm{n}}, \psi_{\text {ATPase }}^{\mathrm{a}}\right)_{1}, \ldots,\left(\psi_{\text {ATPase }}^{\mathrm{n}}, \psi_{\text {ATPase }}^{\mathrm{a}}\right)_{k}\right]$;

Propagate the metabolic model using $\psi$ as input to get updated $p^{+}$at $t_{c}+\Delta t$;

Define $p$ over $\left[t_{c}, t_{c}+\Delta t\right]$ by interpolating linearly between $p_{c}$ and $p^{+}$;

Repropagate electrophysiology model over $\left[t_{c}, t_{c}+\Delta t\right]$ using interpolated $p$ as input;

Update $p_{c}=p^{+}$;

Update $t_{c}=t_{c}+\Delta t$.

end do 


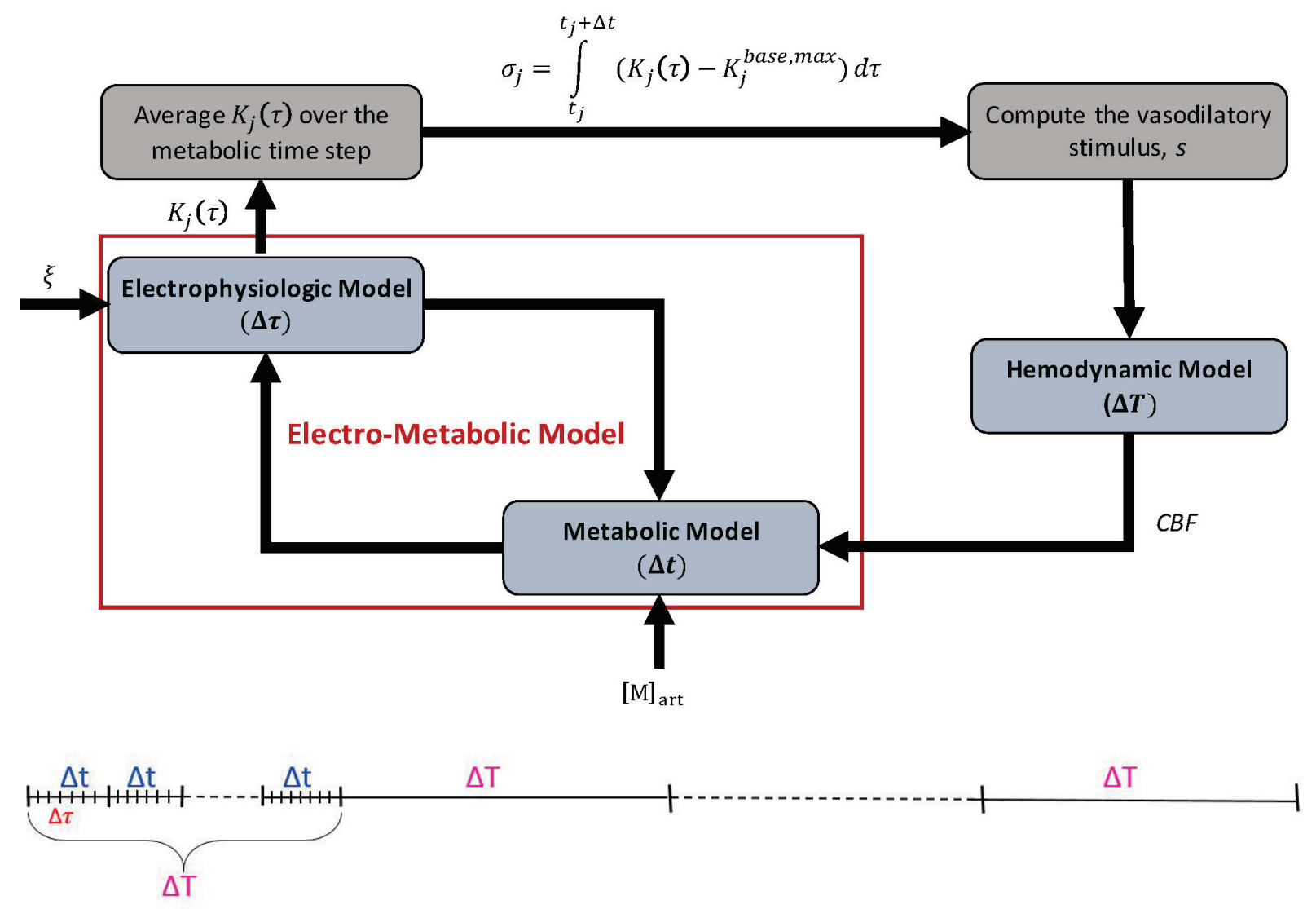

Figure 3: Schematic representation of the three-way feedback loop. The time marching uses three nested time steps, $\Delta T$ for the hemodynamics, $\Delta t$ for metabolism, and $\Delta \tau$ for electrodynamics. The output of the Electro-Metabolic Model over the time interval $\left[t_{j}, t_{j}+\Delta t\right]$ comprises the extracellular potassium profile $K_{j}$, leading to the integrated potassium signal $\sigma_{j}$ and, through integration over the interval $\left[T_{i}, T_{i}+\Delta T\right]$, to the hemodynamic stimulus $s_{i}$ that serves as an input for the hemodynamic model. The hemodynamic model determines the CBF profile that regulates the metabolic model through nutrient supply. The only external inputs are the arterial concentrations of glucose, oxygen and lactate, and the neuronal activation function describing an electrophysiological signal arriving from outside the unit through the presynaptic axon.

\section{Results}

To demonstrate the predicting power of our computational model, we consider two computer experiments. In the first example we track changes in brain metabolism and hemodynamics over an interval of 10 minutes as the system undergoes transitions from an awake resting state, characterized by neuronal mean firing at $8 \mathrm{~Hz}$ frequency to a period of neuronal activation of the duration of 45 seconds, at a mean firing frequency of $90 \mathrm{~Hz}$. In the second one, the duration of the $90 \mathrm{~Hz}$ activation period is increased to 3 minutes, and the total simulation time interval is increased to 30 minutes. The rationale and physiological interpretation of the simulations are discussed in the next section, where we analyze the results in the context of experimental findings reported in the literature. The frequency increase from $8 \mathrm{~Hz}$ to $90 \mathrm{~Hz}$ is induced by a rise of the value of the activation input from $\xi=0.05$ during rest awake to $\xi=2.5$ during neuronal activation. The activation periods are indicated in Figures 4 and 5 by gray shading.

Row A of Figures 4 and 5 shows the time course of metabolite concentrations in blood and the relative increase in 
CBF during the respective activation protocols, and row B the time course of principal metabolite concentrations in the tissue compartments during the simulation period. Row C summarizes the cross-membrane transport rates, while row D and panels (i) and (ii) of row $\mathrm{E}$ the reaction fluxes in neuron and astrocyte. Panels (iii) and (iv) of row E show the time course of the oxygen glucose index (OGI) and the cerebral metabolic rate of glucose. Panels (i) and (iii) of row F show how action potential and frequency, as well as intracellular sodium and extracellular potassium concentrations, change as the system transitions from resting state to activation, and back. Panels (ii) and (iv) of row F show details of the slow after-hyperpolarization. 


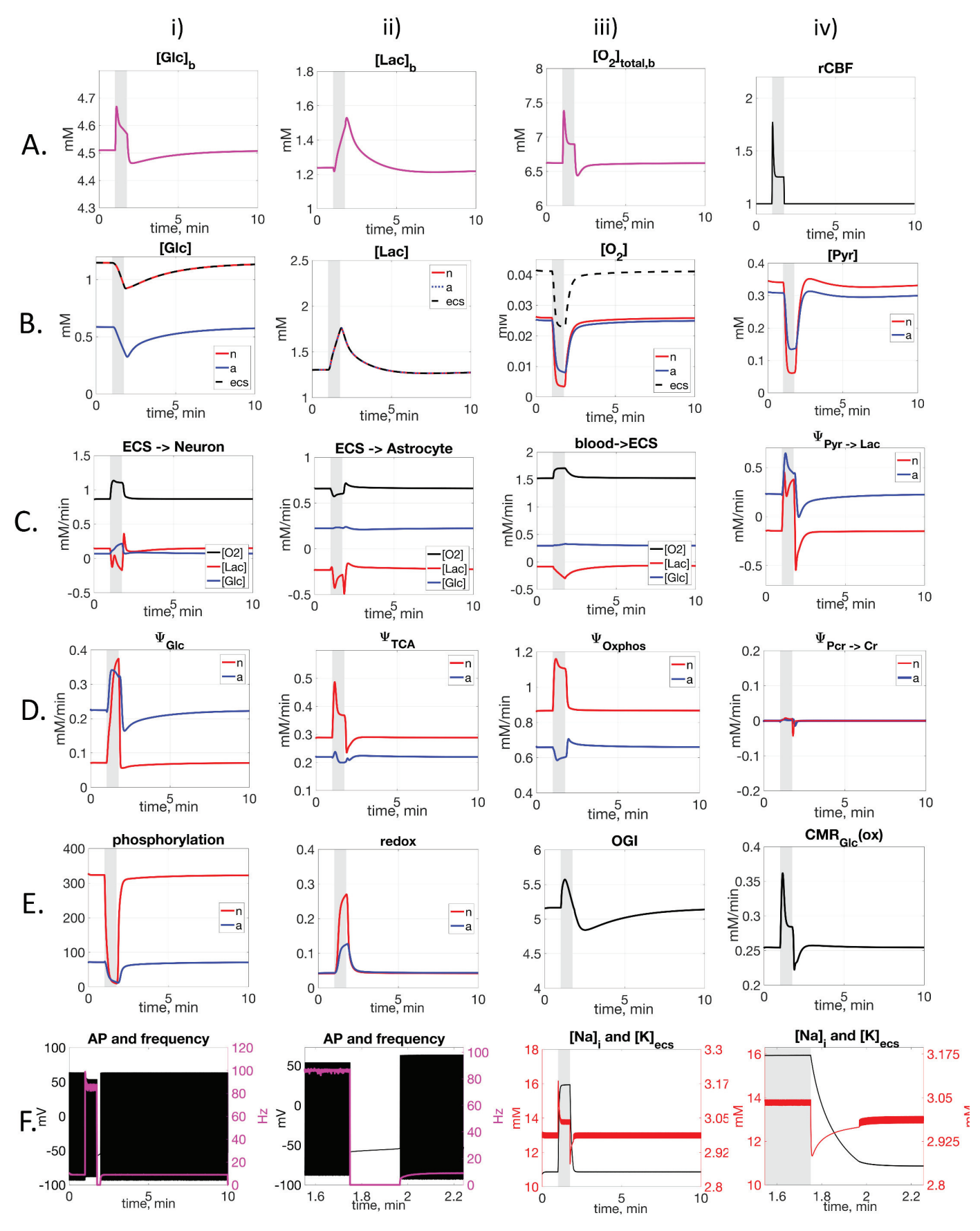

Figure 4: Model predictions of the changes in metabolism, electrophysiology and CBF as the system switches from resting state to high frequency firing for a period of 45 seconds: In each panel, the high activity period is indicated by a gray shadow. Row A. Blood compartment: i) glucose, ii) lactate, iii) oxygen concentrations and iv) relative CBF. Row B: neuron (red), astrocyte (blue) and extracellular space (black) time courses for the concentrations of i) glucose, ii) lactate, iii) oxygen and iv) pyruvate concentrations. Row C: Transport rates between i) extracellular space and neuron, ii) extracellular space and astrocyte, and iii) blood and extracellular space for glucose (blue), lactate (red) and oxygen (black). Panel iv) shows lactate dehydrogenase balance flux for neuron. Row D: Reaction fluxes for i) glycolysis, ii) tricarboxylic acid cycle, iii) oxidative phosphorylation and iv) Creatine phosphorylation net flux. Row E: i) phosphorylation state in neuron (red) and astrocyte (blue), ii) redox state in neuron (red) and astrocyte (blue), iii) oxygen glucose index (OGI), iv) cerebral metabolic rate of glucose oxidation. Row F: i) Action potential and its associated frequency, ii) A detail of the slow after-hyperpolarization, iii) time course of intracellular sodium (black) and extracellular potassium (red), iv) sodium and potassium concentrations in the slow after-hyperpolarization phase. 


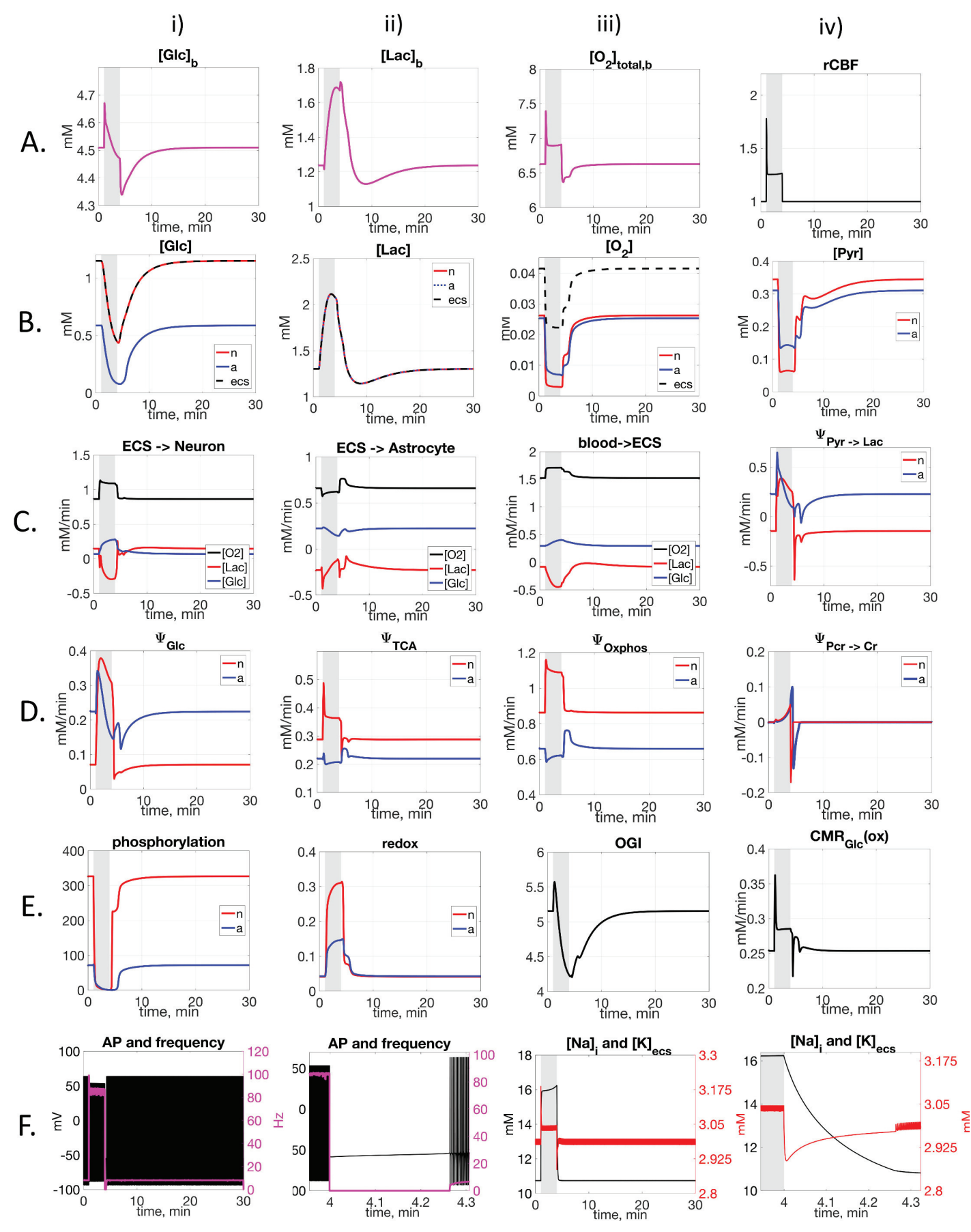

Figure 5: Model predictions of the changes in metabolism, electrophysiology and CBF as the system switches from resting state to high frequency firing for a three-minute period: In each panel, the high activity period is indicated by a gray shadow. Row A. Blood compartment: i) glucose, ii) lactate, iii) oxygen concentrations and iv) relative CBF. Row B: neuron (red), astrocyte (blue) and extracellular space (black) time courses for the concentrations of i) glucose, ii) lactate, iii) oxygen and iv) pyruvate concentrations. Row C: Transport rates between i) extracellular space and neuron, ii) extracellular space and astrocyte, and iii) blood and extracellular space for glucose (blue), lactate (red) and oxygen (black). Panel iv) shows lactate dehydrogenase balance flux for neuron. Row D: Reaction fluxes for i) glycolysis, ii) tricarboxylic acid cycle, iii) oxidative phosphorylation and iv) Creatine phosphorylation net flux. Row E: i) phosphorylation state in neuron (red) and astrocyte (blue), ii) redox state in neuron (red) and astrocyte (blue), iii) oxygen glucose index (OGI), iv) cerebral metabolic rate of glucose oxidation. Row F: i) Action potential and its associated frequency, ii) A detail of the slow after-hyperpolarization, iii) time course of intracellular sodium (black) and extracellular potassium (red), iv) sodium and potassium concentrations in the slow after-hyperpolarization phase. 


\section{Discussion}

A typical neuron population in human cortex consists roughly of principal pyramidal neurons and interneurons, the former ones being more abundant, the interneurons comprising about $10 \%$ of the population, depending on the cortical region $[42,43]$. The firing rate of human cortical neurons vary from one neuron type to another. The fast spiking (FS) neurons generate high frequency action potential trains with little frequency modulation, the maximal mean frequency being about $340 \mathrm{~Hz}$, with momentary instantaneous frequency reaching up to $450 \mathrm{~Hz}$ [44]. Among the interneurons, the FS neurons are abundant, mostly GABAergic neurons, constituting up to $40 \%$ of the neocortical interneuron population. The frequency distribution in an active region of the human brain is location dependent, and difficult to determine. Non-invasive methods, such as EEG and MEG, are mostly believed to observe oscillations of the post-synaptic dipole field [45], which is not directly related to the neuron firing rate. Likewise, clinical depthelectrodes, implanted in the brain of epilepsy surgery patients record local field potentials, which do not translate directly into action potentials. However, it is believed that the high $\gamma$-band (HG) oscillations (60-200 Hz) are related to inhibitory post-synaptic polarization [46], and therefore possibly to FS activity. Further, the HG oscillations are believed to be related to functional activation of the human cortex [47, 48], and to be the representative signature of activated neuronal populations as they describe wide-scale neuronal processes that are associated with cognition and perception [49]. In the simulations of the previous section, we have selected the mean frequency representing high neuronal activity to be $90 \mathrm{~Hz}$; such mean activity could be obtained, e.g., by assuming roughly $90 \%$ of pyramidal cells with firing frequency of $60 \mathrm{~Hz}$, and $10 \%$ of FS interneurons with frequency of $340 \mathrm{~Hz}$. This hypothetical partitioning serves only to justify the parameter selection in our computational model simulation. The durations of the high neuronal activities are chosen to represent an intense task of 45 seconds in the first protocol, while the second protocol, with high intensity firing lasting three minutes, is intended to simulate an extreme event, e.g., a tonic-clonic epileptic episode, that typically continues for 1-3 minutes. Interestingly, the GABAergic interneurons are identified to be susceptible for neuron loss during prolonged epileptic episodes (status epilepticus) of more than 5 minutes [50], supporting the assumption that the high frequency activity using up the energy resources can be

attributed to these neurons. Furthermore, while our current metabolic model is too simplified to distinguish between glutamatergic and GABAergic neurons, the significant role of the latter ones during intensive neuronal activation has been recognized both in experiments [51, 52], as well as in simulation studies [29, 13]. Recall that, according the volume scaling argument, more focal activity can be simulated by scaling the time by the volume fraction, as pointed out in the introduction. However, the combined model proposed here is not directly scalable, as the electrophysiology and metabolism still need to be appropriately coupled [23].

In both computed experiments, the model predicts that within the first second of neuronal activation, CBF increases $77 \%$ above baseline value and 16 seconds after it stabilizes around $26 \%$ above baseline for the duration of the activation period, in agreement with recent literature [53].

We observe a similar spike in the neuronal frequency, which initially reaches $107 \mathrm{~Hz}$ and then rapidly stabilizes at $90 \mathrm{~Hz}$ for the duration of the neuronal activation. In line with what we would expect from electrophysiology alone, there is a reduction in amplitude of the action potential during activation, accompanied by an increase of intracellular sodium and extracellular potassium concentrations.

Remarkably, the model accurately predicts the slow afterhyperpolarization (sAHP) effect, $[54,55]$ a brief neuronal silencing at the end of the activation period lasting approximatively 16 seconds regardless of the duration of the 
preceding activation. Panel (ii) of row (F) of Figures 4 and 5 shows how the activity stops and the membrane is hyperpolarized during the slow AHP period, while panel (iv) in the same row shows the time course of extracellular potassium concentration and intraneuronal sodium concentration during the slow AHP period. The sAHP phenomenon can be interpreted in two ways. As the electrophysiology model is still a single neuron model, the silent period represents a silencing of single neurons after prolonged high activity, however, the time scale for a single neuron may not be realistic. On the other hand, in the context of mean activity of a neuron population, the sAHP represents an average slow-down of the firing activity.

In conjunction with the increase in firing frequency, there is an increase in the consumption of glucose, whose concentration during the activation of three minutes falls to $35 \%$ of its baseline value in neuron and extracellular space, and to $13 \%$ of its initial value in astrocyte, while in the shorter activation simulation, the corresponding lows are $75 \%$ and $50 \%$ of the baseline, respectively. In the blood compartment, following the initial spike in CBF at the beginning of the activation, the glucose concentration experiences an initial increase due to increased availability. This is followed by a slow steady decrease during the activation due to the increased uptake, accompanied by a sharp increase in production of lactate. The lactate concentration in blood increases $40 \%$ over its baseline value during the 45 seconds' activation and $60 \%$ for the activation of 3 minutes; similar increases are observed in lactate levels in the tissue. After the activation ends, the glucose values in blood have sunk below the baseline, more pronouncedly after the longer activation protocol.

There is a significant consumption of pyruvate during neuronal activation, as indicated by the increase in the rate of TCA cycle of $83 \%$ in neuron and 59\% in astrocyte. At baseline, the prevailing direction of lactate dehydrogenase in neuron is slightly towards pyruvate production, but during activation it shifts towards lactate production. In astrocyte, on the other hand, the prevailing direction is slightly towards lactate production at baseline, switching briefly to pyruvate production during the first seconds of activation, followed by a return to lactate production. Thus, the model does not support a strong switch to a lactate supply from astrocyte to neuron during the activation, which has been a topic of active debate in the literature $[56,57]$. To address the question properly, a more comprehensive model would be necessary.

The difference in glucose concentration drops in neuron and in astrocyte is a consequence of the different glucose transporter isoforms in neuron (GLUT3) and astrocyte (GLUT1) which are modeled by different transport rates: The neuronal glucose concentration follows closely to the concentration in the ECS, while a larger concentration gradient is necessary for the glucose transport to astrocyte. The resulting imbalance in glucose partitioning has been identified as one of the key factors for deciding the direction of lactate traffic between the cells [13, 58]. Not surprisingly, during neuronal activation oxygen demand is much higher, and translates into a big decrease in the oxygen levels in tissue: In both activation protocols, the oxygen level in ECS drops by about 40\%, by almost $90 \%$ in neuron, and by $75 \%$ in astrocyte. In the blood compartment we report the total concentration of oxygen, i.e., the sum of the amount of oxygen freely dissolved in plasma and oxygen bound to hemoglobin. An initial spike in oxygen concentration in blood at the beginning of the activation is followed by a modest increase of $3 \%$ over awake resting state value. Once the activation ends, a dip of approximatively $5 \%$ in oxygen blood level occurs, followed by a fast recovery to baseline value.

Similarly, glycolysis in neuron shows a significant increase during neuronal activation, while in astrocyte an initial peak is followed by a decrease below baseline value. During the long activation, this decrease takes place already during the activation, in parallel to the decrease of lactate production by astrocyte. 
In neuron, the rates of the TCA cycle and oxidative phosphorylation reactions show an initial spike after which they settle for the duration of the activation at approximatively $30 \%$ above baseline. In astrocyte these reaction fluxes exhibit a very small decay during activation, followed by a peak at the end of it.

As shown in row $\mathrm{C}$ of Figures 4 and 5, the transport rates between extracellular space and neuron show a significant 4 fold rate increase for glucose and a 30\% increase in oxygen over resting state values. Conversely, there is a big decay in the transfer rate of lactate from extracellular space to neuron during the activation period. In fact, while lactate is transported from extracellular space to neuron at awake resting state, during activation lactate is transported from neuron to extracellular space. The transfer rates of glucose and oxygen from extracellular space to astrocyte exhibit a small decay during activation, matched by a small increase in the rate of lactate efflux from astrocyte to extracellular space. The transport rate of oxygen across BBB shows an increase of $15 \%$, from $1.49 \mathrm{mM} / \mathrm{min}$ during baseline to $1.71 \mathrm{mM} / \mathrm{min}$ during activation. The rate at which glucose crosses the BBB increases about $40 \%$ during activation, while there is a 6 fold increase in the rate at which lactate escapes from ECS to blood. The values predicted by the model at awake resting state and during activation are well within the ranges reported in experimental literature $[59,60]$.

The phosphorylation state in both neuron and astrocyte drops almost to zero by the end of the 3 minutes' neuronal activation period, suggesting that all ATP produced goes towards satisfying the increased energetic demand. Redox state, measured as the ratio between the concentrations of $\mathrm{NADH}$ and $\mathrm{NAD}^{+}$, exhibits a large increase over resting state value during activation: In the 3 minutes' activation protocol, the increases are up to 8 fold in neuron and 4 fold in astrocyte. Panel (iii) in row E of Figures 4 and 5 show the time course of the oxygen glucose index (OGI), defined as the ratio between the cerebral metabolic rate of oxygen and the cerebral metabolic rate of glucose. The resting value 5.2 is in line with the recent studies of OGI in gray matter: Hyder et al. [61] report resting OGI value around 5.3 consistently over the whole brain. During the first 20 seconds of the activation, the OGI index increases about $10 \%$ over baseline, after which it starts to decrease, decreasing to $8 \%$ below the baseline value in the short activation, and $18 \%$ of the baseline value in the long activation, slowly recovering to the resting value after the activation ceases. The decrease during the long activation protocol is well in line with experimental findings [62] for human left primary motor cortex and left supplementary motor cortex when the activation corresponds to several minutes' finger-to-thumb apposition of the right and dominant hand. In the literature, smaller decreases in OGI during moderate have been reported, from $8 \%$ [63] to $6 \%$ [60, 64], which are perfectly in line with our findings in the short activation protocol.

A question of interest from the point of view of, e.g., studying recovery after seizures, is the return times of the metabolite levels to baseline after the activity has ended. In Figure 6, we show the percentage of recovery of the principal metabolites at various time instances for the experiment with an activation period of 45 seconds. Notice that just 1 minute after the neuronal activation has stopped, most of the metabolites have already recovered to $90 \%$ of their baseline values. The astrocytic compartment is significantly slower in terms of recovery compared to the other compartments for most of the metabolites, due to the cleansing role of these cells: Energy is needed for recovering the ion homeostasis after the activation. In astrocyte, one minute after the end of the activation period, glucose has recovered only $75 \%$ of its initial value, oxygen $91 \%$, and the phosphorylation state is at $89 \%$ of its baseline value. 

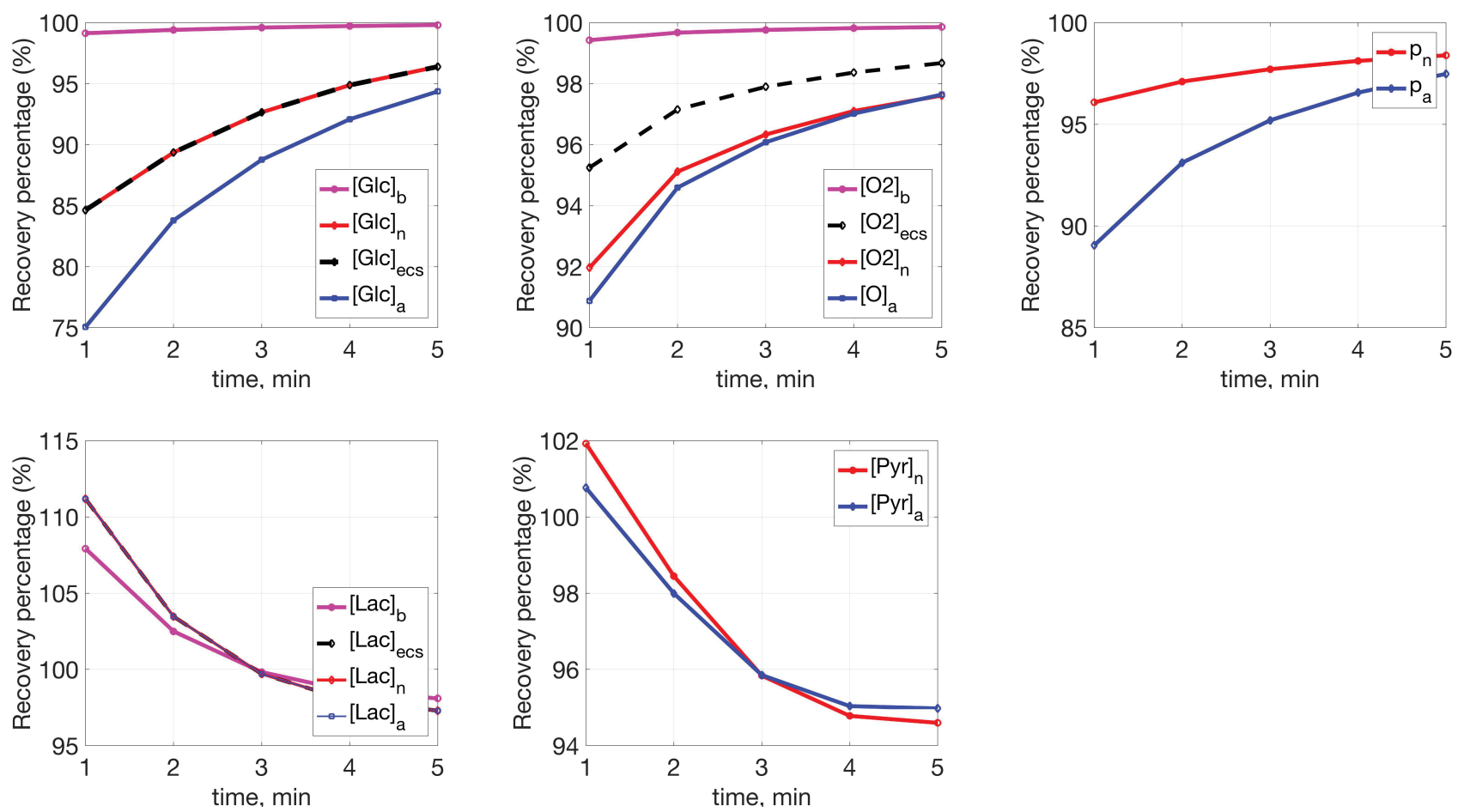

Figure 6: Recovery times required by glucose (first row, left panel), oxygen (first row, center panel), phosphorylation states (first row, right panel), lactate (second row, left panel) and pyruvate (second row, center panel) for the experiment in which the duration of the neuronal activation was 45 seconds. The $x$-axis shows the time after the activation ended in minutes and the $y$-axis shows the recovery of each metabolite, expressed in percentages.

\section{Conclusions}

This paper develops a unified model combining brain energy metabolism with the underlying electrophysiology describing neuron firing and astrocytic ion cleansing, as well as with the metabolite supply system through hemodynamics. The model is based on the idea of two-way mechanisms by which the different constituents communicate with each other: the electrophysiology model and metabolic components exchange information about energy needs and energy availability, the hemodynamic model provides the supply of metabolites and thus controls the energy production, while itself being controlled by the potassium signal originating from neuron firing activity, affecting the vessel compliancies and thus the cerebral blood flow. The numerical simulations demonstrate that even while the metabolic network is rather reduced, we are able to produce model predictions that qualitatively and quantitatively correspond to experimental results.

The integrated model has the potential for interpreting indirect measurements of brain functions. Our understanding of working human brain is largely based on indirect observations of one of the three main components of the model, hemodynamics through BOLD fMRI, metabolism through PET, and electrophysiology through EEG/MEG. While there are good reasons to use one of the modalities as a proxy for another, the proposed integrated model is a step towards putting the implicit assumptions on a well-established basis. Moreover, the model may serve to better understand the mechanisms behind some diseased states that involve more than one of the three aspects of the brain functions. The best candidates are migraine and cortical spreading depression that involve all three aspects of the 
brain, as well as epileptic seizures, which are electrophysiological in nature, but the cell damage is due to energy insufficiency. The connection between epilepsy and metabolism is confirmed by the use of ketogenic diet as seizure control in refractory epilepsy, however, the details remain elusive. In our simulation with a longer activation period, the phosphorylation state going almost to zero causes a quenching of the sodium-potassium pump action which could explain the cell damage and neuronal loss reported following continued seizure-like conditions. While the current model does not contain the elements of describing the damage process, it may serve as a base to study seizure-related cell damage. Observe that in the shorter simulation, while the phosphorylation levels drop, they do not reach as low levels as in the long activation, as one expects.

\section{Acknowledgments}

This work was partly supported by the Basque government through the BERC 2014-2017 and BERC 2018-2021, the Spanish Ministry of Economics and Competitiveness MINECO through the BCAM Severo Ochoa excellence accreditations SEV-2013-0323 and SEV-2017-0718 and the 'Plan Estatal de Investigación. Desarollo e Innovación Orientada a los Retos de la Sociedad' under grants BELEMET (MTM2015-69992-R) and MULTIQUANT (RTI2018093416-B-I00). The work of Daniela Calvetti was partly support by NSF grants DMS-1522334 and NIH grant 1 U01 GM111251-01. The work of Erkki Somersalo was partly support by NSF grants DMS 1714617 and NIH grant 1U01GM111251-01.

\section{Authors contribution statement}

GCR and JP compiled and tested the program and participated in the design of the mathematical model and writing of the article. GCR produced the final results. DC, ES and LGG led the design of the model and participated in the writing of the article. LGG is the chief investigator at BCAM; ES and DC are the chief investigators at CWRU. All authors reviewed and approved the manuscript.

Conflict of interest: The authors declare no duality of interest.

\section{References}

[1] G. A. Dienel, The metabolic trinity, glucose-glycogen-lactate, links astrocytes and neurons in brain energetics, signaling, memory, and gene expression, Neuroscience letters 637 (2017) 18-25.

[2] C. Martin, Contributions and complexities from the use of in vivo animal models to improve understanding of human neuroimaging signals, Frontiers in neuroscience 8 (2014) 211.

[3] M. E. Raichle, M. A. Mintun, Brain work and brain imaging, Annu. Rev. Neurosci. 29 (2006) 449-476.

[4] H. Shibasaki, Human brain mapping: hemodynamic response and electrophysiology, Clinical Neurophysiology 119 (4) (2008) 731-743. 
[5] M. E. Raichle, Circulatory and metabolic correlates of brain function in normal humans, Handbook of Physiology. The Nervous System. Higher Functions of the Brain 1987 (1987) 643.

[6] P. J. Magistretti, I. Allaman, A cellular perspective on brain energy metabolism and functional imaging, Neuron 86 (4) (2015) 883-901.

[7] N. K. Logothetis, J. Pauls, M. Augath, T. Trinath, A. Oeltermann, Neurophysiological investigation of the basis of the fmri signal, Nature 412 (6843) (2001) 150.

[8] D. Attwell, A. M. Buchan, S. Charpak, M. Lauritzen, B. A. MacVicar, E. A. Newman, Glial and neuronal control of brain blood flow, Nature 468 (7321) (2010) 232.

[9] C. Iadecola, The neurovascular unit coming of age: a journey through neurovascular coupling in health and disease, Neuron 96 (1) (2017) 17-42.

[10] M. Bélanger, I. Allaman, P. J. Magistretti, Brain energy metabolism: focus on astrocyte-neuron metabolic cooperation, Cell metabolism 14 (6) (2011) 724-738.

[11] J. Hirrlinger, H. S. Waagepetersen, Brain Energy Metabolism, Springer, 2014.

[12] L. Hertz, L. Peng, G. A. Dienel, Energy metabolism in astrocytes: high rate of oxidative metabolism and spatiotemporal dependence on glycolysis/glycogenolysis, Journal of Cerebral Blood Flow \& Metabolism 27 (2) (2007) 219-249.

[13] D. Calvetti, E. Somersalo, Ménage à trois: the role of neurotransmitters in the energy metabolism of astrocytes, glutamatergic, and gabaergic neurons, Journal of Cerebral Blood Flow \& Metabolism 32 (8) (2012) 1472-1483.

[14] L. K. Bak, A. B. Walls, A. Schousboe, H. S. Waagepetersen, Astrocytic glycogen metabolism in the healthy and diseased brain, Journal of Biological Chemistry (2018) jbc-R117.

[15] L. F. Barros, A. Brown, R. A. Swanson, Glia in brain energy metabolism: A perspective, Glia 66 (6) (2018) $1134-1137$.

[16] T. Takano, G.-F. Tian, W. Peng, N. Lou, W. Libionka, X. Han, M. Nedergaard, Astrocyte-mediated control of cerebral blood flow, Nature neuroscience 9 (2) (2006) 260.

[17] R. Nortley, D. Attwell, Control of brain energy supply by astrocytes, Current opinion in neurobiology 47 (2017) $80-85$.

[18] A. L. Hodgkin, A. F. Huxley, A quantitative description of membrane current and its application to conduction and excitation in nerve, The Journal of physiology 117 (4) (1952) 500-544.

[19] J. R. Cressman, G. Ullah, J. Ziburkus, S. J. Schiff, E. Barreto, The influence of sodium and potassium dynamics on excitability, seizures, and the stability of persistent states: I. single neuron dynamics, Journal of computational neuroscience 26 (2) (2009) 159-170.

[20] J. R. Cressman, G. Ullah, J. Ziburkus, S. J. Schiff, E. Barreto, Erratum to: The influence of sodium and potassium dynamics on excitability, seizures, and the stability of persistent states: I. single neuron dynamics, Journal of computational neuroscience 30 (3) (2011) 781. doi:10.1007/s10827-011-0333-0. 
[21] E. Barreto, J. R. Cressman, Ion concentration dynamics as a mechanism for neuronal bursting, Journal of biological physics 37 (3) (2011) 361-373.

[22] R. Jolivet, J. S. Coggan, I. Allaman, P. J. Magistretti, Multi-timescale modeling of activity-dependent metabolic coupling in the neuron-glia-vasculature ensemble, PLoS computational biology 11 (2) (2015) e1004036.

[23] D. Calvetti, G. Capo-Rangel, L. Gerardo-Giorda, E. Somersalo, A computational model integrating brain electrophysiology and metabolism highlights the key role of extracellular potassium and oxygen, Journal of theoretical biology 446 (2018) 238-258.

[24] D. Calvetti, J. Prezioso, E. Somersalo, Estimating hemodynamic stimulus and blood vessel compliance from cerebral blood flow data, Journal of theoretical biology 460 (2019) 243-261.

[25] J. Prezioso, An inverse problem of cerebral hemodynamics in the bayesian framework, Ph.D. thesis, Case Western Reserve University (2017).

[26] M. J. Barrett, M. H. Tawhai, V. Suresh, Arteries dominate volume changes during brief functional hyperemia: evidence from mathematical modelling, Neuroimage 62 (1) (2012) 482-492.

[27] D. Calvetti, E. Somersalo, Dynamic activation model for a glutamatergic neurovascular unit, Journal of theoretical biology 274 (1) (2011) 12-29.

[28] B. R. Larsen, A. Stoica, N. MacAulay, Managing brain extracellular $\mathrm{K}^{+}$during neuronal activity: the physiological role of the $\mathrm{Na}^{+} / \mathrm{K}^{+}$-ATPase subunit isoforms, Frontiers in physiology 7 (2016) 141.

[29] R. Occhipinti, E. Somersalo, D. Calvetti, Energetics of inhibition: insights with a computational model of the human gabaergic neuron-astrocyte cellular complex, Journal of Cerebral Blood Flow \& Metabolism 30 (11) (2010) 1834-1846.

[30] B. C. Carter, B. P. Bean, Sodium entry during action potentials of mammalian neurons: incomplete inactivation and reduced metabolic efficiency in fast-spiking neurons, Neuron 64 (6) (2009) 898-909.

[31] A. Lajtha, G. Gibson, G. Dienel, Handbook of neurochemistry and molecular neurobiology, Springer, 2007.

[32] M. A. Mintun, A. G. Vlassenko, M. M. Rundle, M. E. Raichle, Increased lactate/pyruvate ratio augments blood flow in physiologically activated human brain, Proceedings of the National Academy of Sciences 101 (2) (2004) 659-664.

[33] J. A. Filosa, A. D. Bonev, S. V. Straub, A. L. Meredith, M. K. Wilkerson, R. W. Aldrich, M. T. Nelson, Local potassium signaling couples neuronal activity to vasodilation in the brain, Nature neuroscience 9 (11) (2006) 1397.

[34] C. F. Lourenço, A. Ledo, R. M. Barbosa, J. Laranjinha, Neurovascular coupling mediated by neuronal derivednitric oxide: Mechanisms in health and dysfunction with impact on aging and Alzheimer's disease (2016) 289-308.

[35] R. B. Buxton, E. C. Wong, L. R. Frank, Dynamics of blood flow and oxygenation changes during brain activation: the balloon model, Magnetic resonance in medicine 39 (6) (1998) 855-864. 
[36] J. B. Mandeville, J. J. Marota, C. Ayata, G. Zaharchuk, M. A. Moskowitz, B. R. Rosen, R. M. Weisskoff, Evidence of a cerebrovascular postarteriole windkessel with delayed compliance, Journal of Cerebral Blood Flow \& Metabolism 19 (6) (1999) 679-689.

[37] Y. Kong, Y. Zheng, D. Johnston, J. Martindale, M. Jones, S. Billings, J. Mayhew, A model of the dynamic relationship between blood flow and volume changes during brain activation, Journal of Cerebral Blood Flow \& Metabolism 24 (12) (2004) 1382-1392.

[38] A. Aubert, R. Costalat, A model of the coupling between brain electrical activity, metabolism, and hemodynamics: application to the interpretation of functional neuroimaging, Neuroimage 17 (3) (2002) 1162-1181.

[39] T. J. Huppert, M. S. Allen, H. Benav, A. Devor, P. Jones, A. Dale, D. A. Boas, A multi-compartment vascular model for inferring arteriole dilation and cerebral metabolic changes during functional activation, Journal of cerebral blood flow and metabolism 27 (6) (2007) 1262.

[40] L. Kocsis, P. Herman, A. Eke, Mathematical model for the estimation of hemodynamic and oxygenation variables by tissue spectroscopy, Journal of theoretical biology 241 (2) (2006) 262-275.

[41] Y. Zheng, D. Johnston, J. Berwick, D. Chen, S. Billings, J. Mayhew, A three-compartment model of the hemodynamic response and oxygen delivery to brain, Neuroimage 28 (4) (2005) 925-939.

[42] T. Freund, G. Buzsáki, Interneurons of the hippocampus, Hippocampus 6 (4) (1996) 347-470.

[43] I. V. Viskontas, A. D. Ekstrom, C. L. Wilson, I. Fried, Characterizing interneuron and pyramidal cells in the human medial temporal lobe in vivo using extracellular recordings, Hippocampus 17 (1) (2007) 49-57.

[44] B. Wang, W. Ke, J. Guang, G. Chen, L. Yin, S. Deng, Q. He, Y. Liu, T. He, R. Zheng, Y. Jiang, X. Zhang, T. Li, G. Luan, H. D. Lu, M. Zhang, X. Zhang, Y. Shu, Firing frequency maxima of fastspiking neurons in human, monkey, and mouse neocortex, Frontiers in Cellular Neuroscience 10 (2016) 239. doi:10.3389/fncel.2016.00239.

[45] M. Hämäläinen, R. Hari, R. J. Ilmoniemi, J. Knuutila, O. V. Lounasmaa, Magnetoencephalography - theory, instrumentation, and applications to noninvasive studies of the working human brain, Rev. Mod. Phys. 65 (1993) 413-497. doi:10.1103/RevModPhys.65.413.

[46] A. Bragin, J. Engel Jr, C. L. Wilson, I. Fried, G. Buzsáki, High-frequency oscillations in human brain, Hippocampus 9 (2) (1999) 137-142.

[47] S. Ray, N. E. Crone, E. Niebur, P. J. Franaszczuk, S. S. Hsiao, Neural correlates of high-gamma oscillations $(60-200 \mathrm{~Hz})$ in macaque local field potentials and their potential implications in electrocorticography, Journal of Neuroscience 28 (45) (2008) 11526-11536. doi:10.1523/JNEUROSCI.2848-08.2008.

[48] M. M. Smith, K. E. Weaver, T. J. Grabowski, R. P. N. Rao, F. Darvas, Non-invasive detection of high gamma band activity during motor imagery, Frontiers in Human Neuroscience 8 (2014) 817. doi:10.3389/fnhum.2014.00817.

[49] D. O. Cheyne, P. Ferrari, Meg studies of motor cortex gamma oscillations: evidence for a gamma 'fingerprint' in the brain?, Frontiers in human neuroscience 7 (2013) 575. 
[50] T. P. Sutula, J. Hagen, A. Pitkänen, Do epileptic seizures damage the brain?, Current opinion in neurology 16 (2) (2003) 189-195.

[51] L. Hertz, J. Drejer, A. Schousboe, Energy metabolism in glutamatergic neurons, gabaergic neurons and astrocytes in primary cultures, Neurochemical research 7 (13) (1988) 605-610.

[52] A. B. Patel, R. A. de Graaf, G. F. Mason, D. L. Rothman, R. G. Shulman, K. L. Behar, The contribution of gaba to glutamate/glutamine cycling and energy metabolism in the rat cortex in vivo, Proceedings of the National Academy of Sciences 102 (15) (2005) 5588-5593. doi:10.1073/pnas.0501703102.

[53] P. Moses, M. DiNino, L. Hernandez, T. T. Liu, Developmental changes in resting and functional cerebral blood flow and their relationship to the bold response, Human brain mapping 35 (7) (2014) 3188-3198.

[54] A. T. Gulledge, S. Dasari, K. Onoue, E. K. Stephens, J. M. Hasse, D. Avesar, A sodium-pump-mediated afterhyperpolarization in pyramidal neurons, Journal of Neuroscience 33 (32) (2013) 13025-13041.

[55] B. King, A. P. Rizwan, H. Asmara, N. C. Heath, J. D. Engbers, S. Dykstra, T. M. Bartoletti, S. Hameed, G. W. Zamponi, R. W. Turner, IKCa channels are a critical determinant of the slow AHP in CA1 pyramidal neurons, Cell reports 11 (2) (2015) 175-182.

[56] C.-P. Chih, E. L. Roberts Jr, Energy substrates for neurons during neural activity: a critical review of the astrocyte-neuron lactate shuttle hypothesis, Journal of Cerebral Blood Flow \& Metabolism 23 (11) (2003) $1263-1281$.

[57] L. Pellerin, P. J. Magistretti, Food for thought: challenging the dogmas, Journal of Cerebral Blood Flow \& Metabolism 23 (11) (2003) 1282-1286.

[58] M. DiNuzzo, S. Mangia, B. Maraviglia, F. Giove, Changes in glucose uptake rather than lactate shuttle take center stage in subserving neuroenergetics: evidence from mathematical modeling, Journal of Cerebral Blood Flow \& Metabolism 30 (3) (2010) 586-602.

[59] P. L. Madsen, R. Linde, S. G. Hasselbalch, O. B. Paulson, N. A. Lassen, Activation-induced resetting of cerebral oxygen and glucose uptake in the rat, Journal of Cerebral Blood Flow \& Metabolism 18 (7) (1998) $742-748$.

[60] F. W. Wehrli, Z. B. Rodgers, V. Jain, M. C. Langham, C. Li, D. J. Licht, J. Magland, Time-resolved MRI oximetry for quantifying CMRO2 and vascular reactivity, Academic radiology 21 (2) (2014) 207-214.

[61] F. Hyder, P. Herman, C. J. Bailey, A. Møller, R. Globinsky, R. K. Fulbright, D. L. Rothman, A. Gjedde, Uniform distributions of glucose oxidation and oxygen extraction in gray matter of normal human brain: no evidence of regional differences of aerobic glycolysis, Journal of Cerebral Blood Flow \& Metabolism 36 (5) (2016) 903-916.

[62] M. S. Vafaee, K. Vang, L. H. Bergersen, A. Gjedde, Oxygen consumption and blood flow coupling in human motor cortex during intense finger tapping: implication for a role of lactate, Journal of Cerebral Blood Flow \& Metabolism 32 (10) (2012) 1859-1868. 
[63] R. G. Shulman, F. Hyder, D. L. Rothman, Insights from neuroenergetics into the interpretation of functional neuroimaging: an alternative empirical model for studying the brain's support of behavior, Journal of Cerebral Blood Flow \& Metabolism 34 (11) (2014) 1721-1735.

[64] R. G. Shulman, F. Hyder, D. L. Rothman, Lactate efflux and the neuroenergetic basis of brain function, NMR in Biomedicine 14 (7-8) (2001) 389-396. 